\title{
Schweizerische Antworten auf Zwangsheiraten als Menschenrechtsverletzung
}

Marianne Schwander

Ein neues Bundesgesetz soll Zwangsheiraten in der Schweiz stärker bekämpfen. Dieses vermag aber insbesondere aus Opfersicht nicht zu befriedigen, da es lückenhaft sowie kriminalpolitisch fragwürdig ist und teilweise eine Verschlechterung der geltenden Rechtslage darstellt. Des Weiteren beschloss der Schweizerische Bundesrat als Ergänzung zum Bundesgesetz für die Jahre 2013 bis 2018 ein Programm mit dem Ziel, die Zusammenarbeit unter Beratungsstellen, Berufsleuten und Schulen zu verstärken sowie Lücken bei Prävention, Beratung, Schutz und Schulung zu schließen. Aus der Sicht der von Zwangsheirat Betroffenen ist diese Ergänzung zu begrüßen. Nicht unproblematisch, möglicherweise sogar kontraproduktiv ist, dass die Thematik Zwangsheirat mit der von Häuslicher Gewalt verknüpft wird.

\section{Einleitung}

Am 15. Juni 2012 verabschiedeten die beiden Kammern des schweizerischen Parlaments das Bundesgesetz über Maßnahmen gegen Zwangsheiraten ${ }^{1}$, welches voraussichtlich am 1. Juli 2013 in Kraft tritt. ${ }^{2}$ Ausgehend von dieser neuen Gesetzgebung befasst sich der Aufsatz nachfolgend mit der Problematik der Zwangsheiraten in der Schweiz und nimmt eine kritische Beurteilung der vom Gesetzgeber beschlossenen und vom Schweizerischen Bundesrat flankierend angeordneten Maßnahmen vor. Dabei wird vergleichend auch die Situation in Deutschland beigezogen.

\section{Zwangsheiraten in der Schweiz: facts and figures}

Eine Zwangsheirat liegt vor, wenn die Ehe gegen den Willen mindestens einer Partei geschlossen wurde, die Weigerung einer Partei kein Gehör fand oder sie es nicht wagte, sich zu widersetzen, weil der innerfamiliäre Druck durch Gewalt, Drohungen oder Nötigung zu groß war. ${ }^{3}$ In Abgrenzung dazu gilt eine Ehe als arrangiert, wenn die Familie zwar die Ehe initiiert und vermittelt hat, das Einverständnis der Betroffenen aber vorliegt. Die betroffenen Personen können bei einer arrangierten Heirat mit ihrem Vetorecht auf die Partnerwahl Einfluss neh- men, dagegen ist bei der erzwungenen Ehe die Ablehnungsmöglichkeit des Heiratsvorschlags versagt respektive nicht gegeben. Auch wenn die Abgrenzung schwierig und der Übergang zwischen einer Zwangsheirat und einer arrangierten Ehe fließend ist, sprechen folgende typischen Phasen für eine arrangierte Ehe: kollektive Partnersuche, familiäre Vorstellungsbesuche, kollektive Entscheidfindung und kollektive Heiratsverhandlungen, aber mit einem Vetorecht. ${ }^{4}$

Es ist schwierig, das Ausmaß der Zwangsheiraten präzise zu beziffern, denn bis anhin gibt es in der Schweiz keine zuverlässigen Statistiken darüber. Eine hierzu im Jahre 2006 verfasste Studie kommt zum Schluss, dass es in der Schweiz ungefähr 17.000 erzwungene Ehen gibt, wobei ein Drittel der Opfer zwischen 13 und 18 Jahre alt und daher unmündig ist. ${ }^{5}$ Eine im Jahre 2012 veröffentlichte Studie, in der die Problematik aus der Perspektive von Expertinnen und Experten in Beobachtungssituationen erhoben wurde, zeigt auf, dass die befragten Institutionen während der letzten zwei Jahre mit 1.391 Fällen konfrontiert waren. ${ }^{6}$

Sowohl Mädchen und Frauen als auch Knaben und Männer werden zwangsverheiratet. In der Schweiz kommen Zwangsheiraten vorwiegend in Einwanderergemeinschaften vor, die jedoch weder einem bestimmten Kulturkreis noch einer bestimmten Religion angehören. Es sind jedoch vornehmlich patriarchalisch strukturierte und traditionalistisch orientierte Gemeinschaften. ${ }^{7}$

\section{Zwangsheirat als}

\section{Menschenrechtsverletzung}

Zwangsheiraten verletzen das Recht auf Ehe, das in der Schweizerischen Bundesverfassung ${ }^{8}$, aber auch in verschiedenen völkerrechtlichen Verträgen als Menschenrecht ${ }^{9}$ verankert ist. Dieses Menschenrecht schützt die Freiheit einer Person im heiratsfähigen Alter, selbst zu entscheiden, ob sie heiraten möchte und wenn ja, wen. Zwangsheiraten verletzen weitere Menschenrechte, beispielsweise das Recht auf sexuelle Selbstbestimmung, auf körperliche und psychische Integrität sowie auf Bewegungsfreiheit. ${ }^{10}$

\section{Gründe für Zwangsheiraten}

Im Zusammenhang mit der Problematik Zwangsheiraten sind die gesellschaftlichen Beziehungen vielschichtig und komplex. Um die Gründe einer traditionsbedingten Menschenrechtsverletzung einordnen $\mathrm{zu}$ können, ist es notwendig, sich mit den historischen, sozialen und kulturellen Hintergründen auseinanderzusetzen. Oft stammen die Betroffenen aus ökonomisch bedrängten Verhältnissen und sind stark in einen familiären Kontext eingebunden. Zudem wird häufig eine schnelle Eheschließung als Disziplinarmaßnahme aufgeführt. In der Regel jedoch basiert in der Praxis eine Zwangsheirat auf einer Verknüpfung verschiedener Gründe. ${ }^{11}$

In einer Befragung von 2006 in Hamburg im Auftrag der Behörde für Soziales, Familie, Gesundheit und Verbraucherschutz zum Thema Zwangsheiraten wurden folgende mögliche Gründe sowie deren Häufigkeit für Zwangsheiraten aufgeführt: Ehre der Familie, Kontrolle über die Kinder, insbesondere die Töchter, sowie Tradition in 44,4 Prozent der Fälle, Versorgung des Kinds sowie finanzielle Motive in 24,1 Prozent der Fälle, Aufenthaltsstatus/Einwanderung in 13 Prozent der Fälle, Familienabsprachen und -verpflichtungen in 13 Prozent der Fälle und in 5,6 Prozent der Fälle wurde als Grund die Religion genannt. ${ }^{12}$

In der Studie findet sich im Fazit u.a. diese für von Zwangsheirat Betroffenen wichtige Aussage: „Nicht hinreichend beantwortet werden konnte die Frage nach den Ursachen, warum einige Zugewanderte in Deutschland ihre Kinder unter Einsatz von psychischer oder physischer Gewalt verheiraten. ... Insgesamt liefert die Erklärung, dass Ansehen und Ehre der Familie gewahrt werden sollen, nur ein unvollständiges Bild. Dieses unvollständige Bild birgt die Gefahr, dass die Diskussion über Zwangsheirat verkürzt z.B. unter der Fragestellung einer mangelnden Integrationsbereitschaft der Zugewanderten geführt wird. Um eine sachliche und lösungsorientierte Diskussion zu führen, wird empfohlen, die Faktoren, die eine Zwangsverheiratung begünstigen, näher zu beleuchten, und dies unter Ein- 
beziehung und Befragung von Zugewanderten. Da das Phänomen Zwangsheirat in Deutschland in erster Linie unter Menschen mit Migrationshintergrund erfolgt, sollten die Strategien für eine Verhinderung auch gemeinsam mit zugewanderten Menschen entwickelt und umgesetzt werden." 13

\section{Der familiäre Kontext}

Insbesondere an unterschiedlichen Vorstellungen zur Selbstbestimmung bei der Partnerwahl entzündet sich ein Generationenkonflikt zwischen den Eltern, in der Regel Migrantinnen und Migranten der ersten Generation, und ihren Kindern. Während die Eltern in einer arrangierten Ehe mit einer Person der gleichen Herkunft eine Garantie für ein sicheres und zufriedenstellendes Leben sehen, ist die Sicht ihrer Kinder, Migrantinnen und Migranten der zweiten oder dritten Generation, grundsätzlich eine andere. Sie sind in der Schweiz oder in einem andern westlich geprägten Land aufgewachsen und sozialisiert worden, ihre Bezugspersonen sind ihre Freundinnen und Freunde sowie Kolleginnen und Kollegen ihres Wohnorts. Sie haben ihre eigenen Vorstellungen von Liebe, Partnerschaft, Selbstbestimmung und Autonomie entwickelt und fühlen sich der ethnischen und familiären Solidarität weniger verpflichtet als ihre Eltern. ${ }^{14}$

In diesem familiären Kontext wählen junge Frauen und Männer, die von ihren Eltern in eine arrangierte Ehe gedrängt oder zwangsverheiratet werden sollen, aus drei unterschiedlichen Bewältigungsstrategien jene aus, die ihnen am ehesten entspricht, wobei im konkreten Fall meist ein Hin und Her zwischen diesen drei Strategien auszumachen ist: die einen setzen auf defensive Abwehr und versuchen, dem familiären Druck zu entfliehen, andere wählen das Mittel der Anpassung und fügen sich dem Druck und wiederum andere reagieren proaktiv und streben Selbstbestimmung an. ${ }^{15}$

Wer die defensive Abwehr wählt, versucht sich dem familiären Druck zu entziehen, indem sie oder er die Freundschafts- und Liebesbeziehung verheimlicht, die Anweisung der Eltern nicht beachtet oder ganz aus der Familie ausbricht. Diese Abwehrstrategie ist für die betroffenen jungen Frauen oder Männer häufig mit belastenden Emotionen, mit Gewalt oder mit dem endgültigen Beziehungsabbruch zu ihrer Familie und ihrer Ethnie verbunden. Wer die direkte Konfrontation mit den Eltern scheut, passt sich an, fügt sich dem Druck des familiären
Kontexts und geht eine Ehe ein, die nicht den eigenen Vorstellungen entspricht. Wer proaktiv reagiert und eine Strategie wählt, die nicht zum Konflikt oder Bruch mit den Eltern führt, den jungen Frauen und Männern aber gleichzeitig ein Festhalten an ihren Wünschen erlaubt, ist auf starke Beziehungen und Unterstützung außerhalb des familiären Kontexts angewiesen. ${ }^{16}$

Unabhängig davon, welche Strategie gewählt wird, sind letztlich die von einer Zwangsheirat bedrohte und betroffenen Personen auf Beratung, Unterstützung sowie auf Information, auch für ihre Eltern, angewiesen und damit auf präventive Maßnahmen. Angewiesen sind sie zugleich auf rechtliche Antworten und damit auf gesetzliche Maßnahmen, die auch den familiären Kontext einbeziehen. Denn die Schweiz ist, wie bereits erwähnt, verpflichtet, bedrohte und betroffene Personen unter anderem auch mit gesetzlichen Maßnahmen gegen Zwangsheiraten zu schützen. ${ }^{17}$

In der erwähnten Befragung wurde auch die Frage gestellt, wie die Handlungsmöglichkeiten zur Verhinderung von Zwangsheiraten verbessert werden können. Die Angaben der Befragten lassen sich in sechs Kategorien unterteilen, die auf unterschiedlichen Ebenen ansetzen: Die Sensibilisierung der Öffentlichkeit für das Thema, die Prävention, die Einbeziehung und die stärkere Teilhabe von Migrantinnen und Migranten, Beratungsangebote für die Betroffenen, deren Unterstützung durch Schutzeinrichtungen und finanzielle Hilfen sowie Änderungen des Ausländergesetzes. ${ }^{18}$

Unter den Ausführungen „Änderungen des Ausländerrechts" finden sich folgende Argumentationen ${ }^{19}$ : Es wird davon abgeraten, bestehende Gesetze zu verschärfen, um Zwangsheiraten zu verhindern, viel wichtiger sei die Aufklärung darüber, was es bedeuten würde, als Frau über das eigene Leben bestimmen zu können. Weiter wird die Problematik rund um die Diskussion über die Anhebung des Zuzugsalters bei Familienzusammenführung auf 21 Jahre genannt. ${ }^{20}$ Die Anhebung würde eher dazu führen, dass Migrantinnen und Migranten sich noch stärker diskriminiert und ausgegrenzt fühlten, so dass der gegenteilige Effekt eintrete und viele Familien sich dadurch noch stärker auf ihre Traditionen zurückziehen würden. Des Weiteren meinten mehrere Befragte, es sollte ein Bleiberecht für Frauen geben, die von Zwangsheiraten betroffen sind, d.h. die Ausländerbehörde sollte Zwangsheiraten als Härtefall in den entsprechenden Vorschriften zum eigenständigen Aufenthaltsrecht bzw. Rückkehrrecht akzeptieren - was der deutsche Gesetzgeber auch verankert hat. ${ }^{21}$

Auch vor diesen Hintergrund sind im Folgenden in einem ersten Schritt die rechtlichen Antworten auf Zwangsheiraten darzulegen, in einem zweiten Schritt insbesondere die strafrechtlichen, wobei jeweils ebenso die Frage beantwortet werden soll, ob diese (straf-)rechtlichen Antworten auch aus Opfersicht überzeugen.

\section{Rechtliche Antworten auf Zwangsheiraten}

Im Jahre 2005 beauftragte in der Schweiz die Staatspolitische Kommission des Nationalrats den Bundesrat, einen Bericht über die „Strafbarkeit von Zwangsheiraten und arrangierten Heiraten “ zu verfassen. ${ }^{22}$ Ein gutes Jahr später überwies der Ständerat eine Motion, worin er den Bundesrat beauftragte, unverzüglich alle notwendigen gesetzgeberischen Maßnahmen zu ergreifen. ${ }^{23}$ Sowohl der Nationalrat als auch der Ständerat stimmten im Jahre 2008 der Streichung des Passus „und arrangierten Heiraten“ zu. Am 23. Februar 2011 hat der Bundesrat die Botschaft zum Bundesgesetz über Maßnahmen gegen Zwangsheiraten verabschiedet, worin er Gesetzesänderungen im Schweizerischen Zivilgesetzbuch $(\mathrm{ZGB})^{24}$, im Bundesgesetz über das Internationale Privatrecht (IPRG) ${ }^{25}$ und im Bundesgesetz über die eingetragene Partnerschaft gleichgeschlechtlicher Paare (PartG) ${ }^{26}$, im Bundesgesetz über die Ausländerinnen und Ausländer $(\mathrm{AuG})^{27}$ und im Asylgesetz $(\mathrm{AsylG})^{28}$ sowie im Schweizerischen Strafgesetzbuch $(\mathrm{StGB})^{29}$ vorschlug. Am 15. Juni 2012 haben der National- und Ständerat das neue Bundesgesetz verabschiedet. ${ }^{30}$

Im Schweizerischen Zivilgesetzbuch ist neu verankert, dass das Zivilstandsamt das Vorliegen von Umständen prüft, die erkennen lassen, dass das Gesuch zum Ehevorbereitungsverfahren offensichtlich nicht dem freien Willen der Brautleute entspricht. ${ }^{31}$ In Ergänzung dazu sind die Zivilstandsbehörden verpflichtet, eine festgestellte Zwangsausübung den Strafverfolgungsbehörden anzuzeigen. ${ }^{32}$

Bereits nach geltendem Recht hat das Zivilstandsamt zu prüfen, ob die Brautleute die Ehe wirklich aus freien Stücken eingehen wollen. Es muss also schon heute die Mitwirkung verweigern, wenn die Ehe nicht dem freien Willen der Brautleute entspricht. 
Um diese Mitwirkung zu versagen, muss neu die Offensichtlichkeit des Zwangs gegeben sein - dies ist aus Opfersicht klar eine Verschlechterung. Zudem ist es problematisch, wenn Zivilstandsbehörden zur Meldung an die Strafverfolgungsbehörden verpflichtet sind und ihnen damit polizeiliche oder ausländerrechtliche Aufgaben übertragen werden.

Des Weiteren wurden die unbefristeten Eheungültigkeitsgründe neu um zwei Tatbestände erweitert: Eine Ehe ist einerseits immer für ungültig zu erklären, wenn sie nicht aus freiem Willen der Brautleute geschlossen wurde, und andererseits wenn die Ehefrau oder der Ehemann noch unmündig ist, es sei denn, die Weiterführung der Ehe entspreche den überwiegenden Interessen des oder der Unmündigen, weil beispielsweise bereits Kinder geboren sind oder die Volljährigkeit kurz bevor steht. ${ }^{33}$ Die Geltendmachung dieser gesetzlichen Ungültigkeitsgründe durch die zuständigen Behörden wurde erleichtert, denn neu sind Behörden des Bundes und der Kantone, die Anlass zur Annahme haben, dass eine ungültige Ehe vorliegt, verpflichtet, diesen Umstand der für die Eheanfechtungsklage zuständigen Behörde zu melden, soweit dies mit ihren Aufgaben vereinbar ist. ${ }^{34}$

Behörden sind, auch nach Jahren, zur Meldung bei der für die Klage zuständige Behörde verpflichtet, wobei die Klage vom Gericht abgewiesen werden sollte, wenn für die zum Zeitpunkt des Eheschlusses Unmündigen die Weiterführung der Ehe ihrem überwiegenden Interesse entspricht. Es stellt sich die Frage, ob diese gesetzgeberische Lösung die Situation der Opfer vor dem Hintergrund des innerfamiliären Drucks nicht zusätzlich verschlimmert, da Gewalt, Drohung und Nötigung während eines Gerichtsverfahrens zunehmen können. Zudem sind mit dieser Regelung weiterhin Minderjährigenehen grundsätzlich zulässig, was aus Opfersicht abzulehnen ist. Denn besonders Minderjährige sind vor Zwangsheiraten zu schützen, da es Aufgabe des Staats ist, das Kindswohl zu gewährleisten.

Die neuen im ZGB verankerten Regelungen gelten auch für die eingetragene Partnerschaft gleichgeschlechtlicher Paare nach Partnerschaftsgesetz. ${ }^{35}$

Im Bundesgesetz über das Internationale Privatrecht wurde eine explizite Regelung der Eheungültigerklärung aufgenommen, um die Anwendung der neuen Eheungültigkeitsgründe im internationalen Verhält- nis zu erleichtern. ${ }^{36}$ Neu ist, dass sämtliche Voraussetzungen für eine Eheschließung in der Schweiz ausschließlich nach schweizerischem Recht zu beurteilen sind. ${ }^{37}$ Zudem ist im Rahmen des IPRG Zwangsheiraten mit einer restriktiveren Haltung gegenüber der Minderjährigenehe entgegenzuwirken und zwar vor dem Hintergrund eines gewandelten Verständnisses des schweizerischen Ordre public. Dies hat zur Konsequenz, dass zum einen in der Schweiz die Eheschließung mit Minderjährigen auch bei ausländischen Brautleuten nicht mehr als zulässig erachtet, und dass zum anderen eine im Ausland geschlossene Minderjährigenehen in der Regel nicht mehr akzeptiert wird $^{38}$ - außer diese ist im überwiegenden Interesse der Eheleute. ${ }^{39}$

Stellvertreterehen sind weiterhin möglich, da der Bundesrat diese als mit dem schweizerischen Ordre public vereinbar erachtet und keinen Handlungsbedarf sieht. ${ }^{40}$ Bei einer Stellvertreterehe müssen die Brautleute für das Zustandekommen der Ehe nicht persönlich anwesend sein, es reicht eine gültige Vollmacht. Hier keinen Handlungsbedarf zu sehen, überzeugt im Zusammenhang mit Zwangsheiraten wiederum aus Opfersicht nicht.

Im Bundesgesetz über die Ausländerinnen und Ausländer sowie im Asylgesetz sind Bestimmungen über den Nachzug eines ausländischen Ehepartners oder einer ausländischen Ehepartnerin ergänzt worden. Haben die zuständigen Behörden bei der Prüfung des Ehepartnernachzugs oder das Bundesamt während des Asylverfahrens Anhaltspunkte dafür, dass für die Ehe ein Ungültigkeitsgrund nach Zivilgesetzbuch vorliegt, so melden sie dies der zuständigen Behörde. Gestützt darauf wird das Gesuch um Ehegattennachzug bis zur Entscheidung der Behörde sistiert. Erhebt die Behörde Klage, so wird das Gesuch bis zum Vorliegen des rechtskräftigen Urteils sistiert. ${ }^{41}$ In Artikel $50 \mathrm{AuG}$ wird neu explizit erwähnt, dass Opfer von Zwangsheirat nach Auflösung der Ehe ein Bleiberecht aus „wichtigen persönlichen Gründen“ in der Schweiz erhalten können. Weitergehende Verbesserungen für Betroffene von Zwangsheirat haben sowohl Bundesrat als auch der National- und Ständerat abgelehnt, beispielsweise, dass Personen, die über eine Aufenthalts- oder Niederlassungsbewilligung in der Schweiz verfügen und ins Ausland zwangsverheiratet werden, über die im Gesetz vorgesehene Frist von in der Regel sechs Monaten hinaus ein Rückkehrrecht haben (Artikel 61
Absatz 2 AuG) ${ }^{42}$ Des Weiteren sind aber auch weitergehende Verschärfungen, beispielsweise den Verzicht auf zusätzliche Einschränkungen beim Familiennachzug, wie das Mindestalter 21 und Sprachkenntnisse, abgelehnt worden. ${ }^{43}$

Zentral ist, dass die von Zwangsheirat Betroffenen die aufenthaltsrechtlichen Konsequenzen nicht einseitig zu tragen haben, der Rechtsanspruch des Bleiberechts aus wichtigen persönlichen Gründen nach Auflösung einer Zwangsheirat tatsächlich umgesetzt wird, aber auch, dass Zwangsheiraten nicht zum Vorwand für die Ausschaffung von Ausländerinnen und Ausländern werden - in der Diskussion im Nationalrat wurde bei der Verankerung eines eigenen Straftatbestands im Strafgesetzbuch die Verknüpfung der Ausschaffung mit der Höhe des Mindestmaßes der Freiheitsstrafe nur knapp abgelehnt. $^{44}$

Im AuG sowie im AsylG sind aus Opfersicht insbesondere zwei Lücken festzumachen: Einerseits eine Regelung analog Deutschland ${ }^{45}$, dass den von den Familien während der Ferien im Ausland zwangsverheirateten Personen ein Rückkehrrecht in die Schweiz über die sechs Monate nach Artikel $61 \mathrm{Ab}$ satz 2 AuG eingeräumt wird und andererseits, dass im Bereich des Asylrechts eine drohende Zwangsheirat als Fluchtgrund anerkannt wird.

Die neuen im AuG sowie im AsylG verankerten Regelungen gelten auch für die eingetragene Partnerschaft gleichgeschlechtlicher Paare nach Partnerschaftsgesetz. ${ }^{46}$

\section{Strafrechtliche Antworten auf Zwangsheiraten im Besonderen}

Im Schweizerischen Strafgesetzbuch ist die Strafbarkeit von Zwangsheiraten neu ausdrücklich verankert. Zwangsheiraten sind als qualifizierter Nötigungstatbestand ausformuliert ${ }^{47}$, was eine höhere Strafdrohung mit Freiheitsstrafe bis zu fünf Jahren oder Geldstrafe bedeutet (neu Abs. 1 von Art. 181a StGB). Strafbar wird auch, wer die Tat im Ausland begeht, falls diese auch am Begehungsort strafbar ist und er oder sie sich in der Schweiz befindet (neu Abs. 2 von Art. 181a StGB).

Der Antrag für ein obligatorisches Strafminimum von zwei Jahren ist im Nationalrat nur knapp mit 95 gegen 86 Stimmen abgelehnt worden. ${ }^{48}$ Ziel eines Mindestmaßes von zwei Jahren Freiheitsstrafe war es, die Ausschaffung von ausländischen Personen zu erreichen, die sich der Nötigung schul- 
dig machten. ${ }^{49}$ Nach Auffassung der Nationalräte und Nationalrätinnen, die diesen Antrag unterstützten, sollte das Strafrecht daher unabhängig vom Unrechtsgehalt der konkreten Tat dazu dienen, ausländische Personen auszuschaffen respektive mit dem Instrument des Strafrechts sollte das AuG verschärft werden. Eine entsprechende Regelung hätte nicht nur zur Folge gehabt, dass verurteilte ausländische Personen aus der Schweiz hätten ausgewiesen werden können, sondern Opfer würden sich noch weniger an den Staat wenden, wenn der Familie dadurch die Ausschaffung drohen würde. Die Festlegung eines Mindestmaßes von zwei Jahren Freiheitsstrafe hätte mehr symbolischen Charakter als Hilfe für die von Zwangsheirat betroffenen Opfer bedeutet - ungeachtet der Niederlage im Nationalrat hält eine Minderheit an ihrer Forderung fest: Ein paar Tage nach der Debatte reichte ein Parlamentarier der Christlichdemokratischen Volkspartei CVP eine Motion mit dem Titel „Straftatbestand der Zwangsheirat als Ausschaffungsgrund“ ein. ${ }^{50}$

Die Frage, ob Zwangsheiraten überhaupt explizit im StGB verankert werden sollen oder nicht, wurde in den politischen Gremien kontrovers diskutiert.

Im Jahre 2007 bezog der Bundesrat in einem Bericht „Strafbarkeit von Zwangsheiraten und arrangierten Heiraten“ zur Frage, ob sich ein neuer Straftatbestand aufdränge, wie folgt Stellung:

„Das Strafgesetzbuch enthält zwar keine ausdrückliche Bestimmung, die erzwungene Heiraten unter Strafe stellt. Erzwungene Heiraten werden jedoch durch den Tatbestand der Nötigung erfasst, von Amtes wegen verfolgt und mit Freiheitsstrafe bis zu drei Jahren oder Geldstrafe bestraft. Die ausdrückliche Erwähnung der Zwangsheirat als Fall einer schweren Nötigung oder die Einführung einer neuen Strafnorm ,Zwangsheirat' könnte zwar das Problembewusstsein in der Öffentlichkeit schärfen. Es ist jedoch zweifelhaft, ob dieses Signal Täter und Opfer überhaupt erreichen würde. Zudem würden die bisherigen Probleme bei der Aufklärung des Sachverhalts kaum gelöst. " 51

Interessant ist, dass, knapp zwei Jahre später, im Rahmen der Vernehmlassung, die Meinungen zur Frage, ob im StGB explizit ein Artikel gegen Zwangsheiraten zu verankern sei, stark auseinander gingen: Eine Mehrheit der Kantone, die Grüne Partei und eine Reihe von Organisationen wollten auf eine Änderung des StGB entsprechend dem Vorschlag des Bundesrats verzichten, d.h. keine Verschärfung des StGB und damit keine Verankerung eines neuen Artikels 181a StGB. Gutgeheißen wurde der ausdrückliche Verzicht insbesondere, weil eine solche Norm einerseits unnötig sei und in der Praxis wohl kaum in Anspruch genommen würde ${ }^{52}$ und andererseits nicht von der Hand zu weisen sei, „dass diese in erster Linie missbräuchlich zur generellen Diskreditierung ausländischer Gruppen und zur Beschneidung legitim beanspruchter Grundrechte benutzt werden "53. Hingegen sprachen sich etliche Kantone sowie verschiedene Parteien und Organisationen für eine Qualifizierung als schwere Nötigung oder für die Einführung einer neuen Strafnorm aus. ${ }^{54}$ Begründet wurde die Aufnahme damit, eine Zwangsheirat habe einerseits besonders gravierende Folgen für das Opfer und es rechtfertige sich daher, diese Verletzung explizit im StGB zu verankern und andererseits wurde argumentiert, andere Länder, u.a. Deutschland, hätten den Tatbestand der Zwangsheirat in den Katalog der besonders schweren Nötigungsfälle eingefügt. ${ }^{55}$

Gestützt darauf hat der Bundesrat das Eidgenössische Justiz- und Polizeidepartement beauftragt, bei der Ausarbeitung der Botschaft zum Bundesgesetz über Maßnahmen gegen Zwangsheiraten eine Verstärkung des strafrechtlichen Schutzes vorzusehen ${ }^{56}$ und dieser ist, wie bereits erwähnt, nun neu im StGB explizit verankert.

Vor diesem Hintergrund stellt sich die Frage, warum die Diskussion über eine Verankerung eines eigenständigen Straftatbestands gegen Zwangsheiraten ins StGB so kontrovers geführt wird respektive wer oder was im Zentrum dieser Debatte steht: Die von Zwangsheiraten Betroffenen oder eine bestimmte Ausländerpolitik. Diese Frage kann sowohl aus einem gesellschaftspolitischen als auch aus einem kriminalpolitischen Blickwinkel beantwortet werden.

\section{Gesellschaftspolitische und krimi- nalpolitische Veränderungen}

Für die gegenwärtigen, seit längerem beobachtbaren Veränderungen, diejenigen der Globalisierung und Ökonomisierung - auch der Staaten - sowie die individuellen Verunsicherungen, sind in Staat und Gesellschaft weder im sozialen noch im rechtlichen Normensystem angemessene Antworten gefunden worden. Grundfesten persönlicher Orientierungen werden in Frage gestellt und erschüttert. ${ }^{57}$ Das Gefühl genereller Vulnerabilität, fehlender Kontrollierbarkeit und der Undurchschaubarkeit erweist sich als kollektives Muster, welches Grundlage für diffuse Ängste und Projektionen bildet. Derartige Lebensängste und Unsicherheitsgefühle finden in der Verbrechensfurcht eine fassbare und damit politisch instrumentalisierbare Ausprägung. Dies vermag den Aufschwung der Verbrechensfurcht von einem lokal begrenzten Phänomen zu einem zentralen gesellschaftlichen Problem zu erklären. Als solches ist die Verbrechensfurcht auch für die Kriminalpolitik bedeutsam, hat sie doch die reale Konsequenz, dass sie sich auf das Ausmaß des Sicherheits- und Strafbedürfnisses der Bevölkerung auswirkt. ${ }^{58}$

Die Politiker und Politikerinnen nehmen sich des Themas ohne zu zögern an und versuchen, beispielsweise mit mehr und härterem Strafrecht, wenn nicht mehr Sicherheit, so doch mehr Sicherheitsgefühle zu produzieren. Indem Kriminalität in ihrer Konzentration eine Vielzahl gesellschaftlicher Missstände und Problemlagen darstellt, eignet sich eine Herangehensweise an singuläre Kriminalitätserscheinungen, statt an komplexe strukturelle Probleme, hervorragend zur politischen Profilierung. Mit dem Ruf nach harten Strafen und einem konsequenten Vorgehen gegen kriminelle Personen und Gruppen erfolgt Exklusion nunmehr als ein demonstratives moralisches Bekenntnis ${ }^{59}$ - die Forderung, ausländische Personen auszuschaffen, die sich der Zwangsheirat schuldig gemacht haben, heißen verschiedenste Bevölkerungsgruppen gut: Einerseits sind dies politische Parteien und Bevölkerungsgruppen, die mit ausländerfeindlichen Parolen Politik betreiben, andererseits aber auch politische Gruppierungen, die sich der Problematik Gewalt gegen Frauen annehmen, im Endeffekt aber - unbewusst oder bewusst - helfen, antimuslimische und immigrantenfeindliche Tendenzen zu unterstützen.

Ob Opfer von Zwangsheiraten durch eine Verschärfung im Strafrecht profitieren, ist mehr als fraglich, weil bereits heute die Möglichkeit besteht, Strafanzeige wegen Nötigung zu erstatten. Der neue qualifizierte Straftatbestand ist daher unnötig, denn eine Verschärfung von Gesetzen und damit auch des Strafrechts ist nicht im Interesse der von Zwangsheirat Betroffenen. Zudem ist eine Verschärfung auch kriminalpolitisch fragwürdig, da Erfahrungen im Ausland zeigen, dass die Opfer sich zwar Schutz und Hilfe vor Zwangsheiraten wünschen, aber die 
Täter und Täterinnen, die in der Regel nahe Verwandte sind, nicht ins Gefängnis bringen wollen. Die Opfer setzen alles daran, in irgendeiner Form die Beziehung zur Familie aufrecht zu erhalten - ein repressiveres Strafrecht entspricht daher nicht ihrem Interesse.

Strafrecht ist ein unverzichtbares Element einer modernen Gesellschaft, ohne Strafrecht gibt es diese nicht. Heute wird Strafrecht aber eher als prima ratio oder gar als sola ratio und nicht mehr als ultima ratio angesehen. Werden jedoch immer mehr gesellschaftliche Aufgaben dem Strafrecht überwiesen, ist dies zum Scheitern verurteilt. Denn Gesellschaftspolitik, die sich auf Strafrecht konzentriert oder gar beschränkt, ist zum Misslingen verurteilt. ${ }^{60}$ Die Problematik Zwangsheiraten ist daher aus einem Blickwinkel der davon Betroffenen zu regeln, sei dies mit Beratung, Unterstützung und Prävention, in die auch die Eltern einzubeziehen sind, sei dies aber auch durch gesetzliche Maßnahmen, nicht aber mit Verschärfungen im Strafrecht.

\section{Flankierende Maßnahmen zum neuen Bundesgesetz}

Am 14. September 2012 beschloss der Bundesrat als flankierende Maßnahme zum neuen Bundesgesetz über Maßnahmen gegen Zwangsheiraten, ein fünfjähriges Programm mit dem Ziel, die Zusammenarbeit unter Beratungsstellen, Berufsleuten und Schulen zu verstärken und Lücken bei Prävention, Beratung, Schutz und Schulung zu schließen. Das Programm sieht vor, dass innerhalb von fünf Jahren in allen Regionen funktionierende „Netzwerke gegen Zwangsheirat“ entstehen, in denen Lehrpersonen, Berufsleute und Beratungsstellen im Bereich Häusliche Gewalt und Integration zusammenarbeiten und sich regelmäßig austauschen. Ziel ist es, über die verbesserte Zusammenarbeit dafür zu sorgen, dass für die unterschiedlichen Kategorien möglicher Opfer und zwangsausübenden Personen Angebote und Maßnahmen zur Prävention entwickelt werden. Opfer sollen zudem auf bessere Beratung und größeren Schutz zählen können, in dem für sie beispielsweise Plätze in Frauenhäusern zur Verfügung gestellt werden. Der Bundesrat setzt in den Jahren 2013 bis 2018 insgesamt zwei Millionen Franken für das Programm „Bekämpfung Zwangsheirat“ ein. ${ }^{61}$

Das Programm „Bekämpfung Zwangsheirat" stützt sich auf die Untersuchung „Zwangsheiraten in der Schweiz: Ursa- chen, Formen, Ausmaß“, die das „Maison d'analyse des processus sociaux" MAPS der Universität Neuenburg im Auftrag des Bundes erstellt hat. ${ }^{62}$

Aus der Sicht der von Zwangsheirat Bedrohten und Betroffenen ist das Programm des Bundesrats klar zu begrüßen, insbesondere auch als Ergänzung des Bundesgesetzes über Maßnahmen gegen Zwangsheiraten. Auch zu unterstützen ist, dass Untersuchungen zur qualitativen Erfassung der von Zwangsheiraten Betroffenen in der Schweiz durchgeführt werden. Nicht unproblematisch, sogar kontraproduktiv einzustufen ist jedoch, dass in dieser Untersuchung und daher auch im Programm des Bundesrats, die Problematiken Zwangsheiraten und Häusliche Gewalt zusammen verknüpft werden.

Im Folgenden sind insbesondere die Verknüpfung von Zwangsheiraten und Häuslicher Gewalt, aber auch kulturalisierende Konnotationen zu Zwangsheiraten zu diskutieren.

\section{Kritik an der Diskussion über Zwangsheiraten}

Nicht allein in der öffentlichen Auseinandersetzung, sondern, wie bereits erwähnt, auch in wissenschaftlichen Untersuchungen geht die Diskussion über Zwangsheiraten relativ häufig Hand in Hand mit der Diskussion über Häusliche Gewalt. Die Debatte tendiert in diesem Zusammenhang zu einer Fokussierung auf einzelne Phänomene, die eine Zuschreibung bestimmter Rollenbilder oder Geschlechterverhältnisse und Pauschalverurteilungen der Familien- und Ehekonzepte von Migrantinnen und Migranten beinhaltet. ${ }^{63}$ So fasst u.a. Westphal die Problematik Geschlechterstereotype und Migration wie folgt zusammen:

„Die öffentliche Auseinandersetzung um Zwangsverheiratung, Ehrenmorde und zunehmende Verhüllung von Mädchen und Frauen muslimischer Herkunft aktualisiert die Debatte um Migration und Geschlecht. Sie belebt in erstaunlicher Weise die Annahme, in Migrantenfamilien herrschten traditionelle Verhältnisse, die vor allem durch patriarchal-autoritäre Beziehungen zwischen den Geschlechtern und den Generationen bestimmt seien. Entweder wird den Migrantinnen und Migranten ein Festhalten bzw. eine Konservierung traditioneller Werte und Beziehungsmuster oder eine Art Re- bzw. Neotraditionalisierung unterstellt. Im Ergebnis werden beide als bedrohliche Rückzugs- und Desintegrationstendenzen wahrgenommen. Diese Wahrnehmungsmuster im Kontext von Migration, Kultur und Geschlecht sind nicht neu. Es ist grundsätzlich stark vereinfachend. Insgesamt sind Pluralität und Differenziertheit in den Lebenszusammenhängen von Migrantenfamilien sowie stattfindende Neuinterpretationen der Geschlechterverhältnisse und Familienstrukturen in Rechnung zu stellen. Die mit dieser Vereinfachung einhergehende Pauschalisierung erschwert und verhindert eine solide fachliche Diskussion und Einordnung des Phänomens der Zwangsverheiratung in Deutschland. Erschwerend kommt hinzu, dass empirische Untersuchungen über Entwicklung, Verläufe und Ausmaß noch weitgehend fehlen. Deutlich ist, dass Zwangsverheiratung keineswegs das Hauptproblem von Migrantinnen und Migranten darstellt." 64

Auch wenn Zwangsheiraten bezogen auf Personen mit Migrationshintergrund diskutiert werden müssen und Zwangsheiraten eine Form von Gewalt darstellen, betrifft Häusliche Gewalt keineswegs ausschließlich Migrantinnen und Migranten. Zahlen der Basler Polizei und eine Untersuchung aus dem Kanton Zürich belegen zwar, dass bei Häuslicher Gewalt Migrantenfamilien statistisch übervertreten sind. ${ }^{65}$ Eine weitere Untersuchung aus der Stadt Zürich zeigte jedoch auf, dass Migrantenfamilien, in denen Häusliche Gewalt vorkommt, insgesamt unter größeren Stressfaktoren leben. ${ }^{66}$

Im Jahre 1895 veröffentlichte der Jurist Albert Meyer - er wurde später Bundesrat eine methodisch herausragende Arbeit über die Kriminalität von Migranten im Kanton Zürich. Er wies empirisch nach, dass die Zugewanderten, die damals 26 Prozent der Bevölkerung ausmachten, in den Verurteilungszahlen, vor allem bei der Gewaltkriminalität, mit 51 Prozent vertreten waren. Nur waren diese Zugewanderten nicht Ausländerinnen und Ausländer, sondern Schweizer Bürgerinnen und Bürger aus andern Kantonen, vor allem aus der Ost- und Innerschweiz, die im Rahmen der schweizerischen Binnen-Migration vom Land in die Großstadt gezogen waren und dort in diskriminierten Quartieren und benachteiligten Verhältnissen lebten. ${ }^{67}$

Werden die beiden Problematiken Zwangsheirat und Häusliche Gewalt einander gegenüber gestellt oder miteinander verglichen, ist festzuhalten, dass bei der Häuslichen Gewalt die Bedrohung in der Regel von einer Person ausgeht, bei Zwangsheiraten dagegen von mehreren. Häusliche 
Gewalt basiert auf einem individuellen, Zwangsheiraten auf einem normativen und kollektiven Charakter. Bei Häuslicher Gewalt sind in rund 80 Prozent der Fälle Frauen, bei Zwangsheiraten sind beide Geschlechter betroffen. Zudem sind in rund 80 Prozent der Fälle von Häuslicher Gewalt Männer Täter, bei Zwangsheiraten sind hingegen sowohl Frauen als auch Männer Täterinnen und Täter. ${ }^{68}$

Aus Opfersicht ist es wichtig, dass diese beiden Problematiken und damit auch deren Opfer für sich je ernst genommen werden, damit den jeweilig davon Bedrohten und Betroffenen auch tatsächlich geholfen werden kann, dies insbesondere auch, weil die Antworten auf Zwangsheiraten und Häusliche Gewalt nicht die gleichen sind respektive nicht sein können.

Ein weiterer Aspekt im Zusammenhang mit Zwangsheiraten bezieht sich auf kulturalisierende Konnotationen: Während in älteren Debatten und früheren Untersuchungen der Blick entweder in erster Linie auf Kindsverheiratungen oder auf Fragen wie Eheverhalten von Migrantinnen und Migranten und transnationalen EheschlieBungen lag, wird in den letzten Jahren vielfach ein interner Zusammenhang zwischen Zwangsheiraten und Schlagwörtern wie Islam, Ehre und Kultur hergestellt: Gewalt wird vor diesem Hintergrund traditionsbedingt interpretiert und in der Kultur, Ehre und Religion bestimmter Migrantengemeinschaften verortet, wobei Kultur in der Regel auf nationale oder auch ethnische Herkunft reduziert und mit bestimmten Charakterzügen belegt wird. Damit wird auch eine Verknüpfung von Sexismus und Gewaltbereitschaft von Migrantinnen und Migranten vorgenommen $^{69}$ - und das nicht nur in der politischen Debatte. Dieser Blickwinkel findet sich insbesondere auch in Publikationen, beispielsweise von Ayaan Hirsi-Ali ${ }^{70}$ oder Necla Kelek ${ }^{71}$ sowie in Stellungnahmen von Alice Schwarzer, in denen die Problematik Gewalt gegen Frauen im Endeffekt als antimuslimische und immigrantenfeindliche Tendenz ausgelegt werden muss. ${ }^{72}$

In der Untersuchung von Mirbach/Schaak/ Triebl wurden von befragten Einrichtungen vor allem die Befürchtung einer kulturalisierenden Perspektive und einer einseitigen Zuschreibung von Gewaltphänomenen auf Personen mit Migrationshintergrund benannt:

„Es besteht die Notwendigkeit von Schutz und Unterstützung für von direkter per- sonaler Gewalt betroffenen Mädchen und Frauen. Dies wird inzwischen gesellschaftlich anerkannt und politisch gefördert. Problematisch erscheint uns die Thematik auf der diskursiv geführten Ebene, bei der die Anerkennung der strukturellen Verankerung von Gewalt in hierarchische Geschlechterverhältnisse meist ausgeblendet wird. Durch die Fokussierung auf Gewalt gegen Mädchen und Frauen mit Migrationshintergrund sowie Zwangsverheiratung und Ehrenmorde, besteht die Gefahr der Instrumentalisierung der Betroffenen und fördert die Abgrenzung von Mehrheitsgesellschaft." 73

„Durch die sehr einseitige Darstellung in den Medien und die Gleichstellung von arrangierter Ehe mit Zwangsheirat der selbsternannten türkischen Expertinnen hat sich eine Voreingenommenheit muslimischer Eltern gegenüber entwickelt, die wir sowohl in der deutschen Bevölkerung als auch in der ,Fachwelt' antreffen. Hierdurch kommt es allgemein zu der Einschätzung, Islam und Zwangsehe gehören zusammen, wodurch die Vorbehalte den Muslimen gegenüber ,berechtigterweise' verstärkt werden. ... die öffentliche Fokussierung auf die Muslime ist kontraproduktiv, bzw. schafft neue Probleme. “74

Abschließend soll die Situation der Zwangsheirat, wie sie in Deutschland gesetzlich geregelt ist, zusammenfassend und vergleichend dargelegt werden. ${ }^{75}$

\section{Deutschland}

Das Gesetz zur Bekämpfung der Zwangsheirat und zum besseren Schutz der Opfer von Zwangsheirat sowie zur Änderung weiterer aufenthalts- und asylrechtlicher Vorschriften (ZwHeiratBekG) ist in Deutschland am 1. Juli 2011 in Kraft getreten. ${ }^{76}$

Im Strafgesetzbuch wurde ein eigenständiger Straftatbestand gegen Zwangsheirat geschaffen, wobei die Nötigung zur Eingehung einer Ehe mit einer Freiheitsstrafe von sechs Monaten bis zu fünf Jahren bestraft wird. Bestraft wird ebenso, wer das Opfer in ein Gebiet außerhalb von Deutschland bringt oder davon abhält, von dort zurückzukehren. $^{77}$

Da nach Meinung vieler im Falle einer drohenden Zwangsheirat eher selten eine Anzeige gegen die eigenen Eltern eingereicht wird, habe die Einführung eines eigenständigen Straftatbestands sowie die Heraufsetzung des Strafmaßes eher einen abschreckenden Charakter für die Familie, bringe jedoch den von Zwangsheirat Betroffenen wenig. Wichtig sei jedoch, dass auch eine im Ausland durchgeführte Zwangsheirat strafrechtlich verfolgt werden könne. ${ }^{78}$

Dagegen ist die Schaffung eines eigenständigen Wiederkehrrechts für die ausländischen Opfer von Zwangsheirat, die von der Rückkehr nach Deutschland abgehalten werden, von zentraler Bedeutung. Das eigenständige Wiederkehrrecht gilt innerhalb von drei Monaten nach Wegfall der Zwangslage und ist spätestens bis vor Ablauf von fünf Jahren geltend zu machen. Hat sich die ausländische Person vor ihrer Ausreise acht Jahre rechtmäßig in Deutschland aufgehalten und sechs Jahre eine Schule in Deutschland besucht, erhöht sich das Wiederkehrrecht auf zehn Jahre. ${ }^{79}$

Des Weiteren ist die Antragsfrist zur Aufhebung der Ehe im Bürgerlichen Gesetzbuch von einem auf drei Jahre verlängert worden und zwar zwecks Verminderung des Anreizes zur Eingehung einer Scheinehe. ${ }^{80}$ Bei der Erhöhung der Ehebestandszeit auf drei Jahre besteht nun jedoch die Gefahr, dass durch diese Regelung die Abhängigkeit der Opfer von Zwangsheiraten von ihrem Ehepartner erhöht wird.

Ins Aufenthaltsgesetz sind drei weitere Bestimmungen aufgenommen worden: Eine Ausländerbehörde ist explizit verpflichtet, vor Verlängerung einer Aufenthaltserlaubnis festzustellen, ob die ausländische Person ihrer Pflicht zur Integrationsteilnahme nachgekommen ist. ${ }^{81}$ Daneben sind Datenübermittlungsregelungen im Zusammenhang mit der Durchführung von Integrationsmaßnahmen, die in der Integrationsverordnung enthalten sind, neu im Aufenthaltsgesetz geregelt. ${ }^{82}$ Im Interesse von Asylbewerberinnen und Asylbewerbern und geduldeten Personen wird für diese Personengruppen die Möglichkeit einer Ausnahme von der räumlichen Beschränkung in Fällen der Ausübung einer Beschäftigung, des Schulbesuchs, der Ausbildung und des Studiums geschaffen. ${ }^{83}$

Aus Opfersicht kann festgehalten werden, dass insbesondere die Verankerung des Rückkehrrechts von bis zu fünf Jahren respektive von bis zu zehn Jahren gutzuheißen, dagegen die Erhöhung der Ehebestandszeit abzulehnen ist. Ersteres kann auch vergleichend mit der neuen Lösung in der Schweiz als Vorteil genannt werden, zweiteres als Nachteil. In beide Strafgesetzgebungen sind Verschärfungen aufgenommen worden, was aus Opfersicht klar abzulehnen ist. 


\section{Fußnoten:}

1 Gesetzestext siehe BBl 20125937.

2 Nach telefonischer Auskunft des Bundesamts für Justiz.

3 „Zwangsverheiratungen" liegen dann vor, wenn mindestens einer der Eheleute durch die Ausübung von Gewalt oder durch die Drohung mit einem empfindlichen Übel zum Eingehen einer formellen oder informellen (also durch eine religiöse oder soziale Zeremonie geschlossenen) Ehe gezwungen wird und mit seiner Weigerung kein Gehör findet oder es nicht wagt, sich zu widersetzen. "Mirbach Thomas/Schaak Torsten/Triebl Katrin, Zwangsverheiratung in Deutschland. Anzahl und Analyse von Beratungsfällen, Opladen/Berlin/Farmington Hills 2011, S. 13.

4 Strafbarkeit von Zwangsheiraten und arrangierten Heiraten: Bericht des Bundesrates in Erfüllung des Postulates 05.3477 der Staatspolitischen Kommission des Nationalrates vom 9.9.2005, S. 8 f. Zu Anzahl und Analyse von Beratungsfällen in Deutschland siehe Mirbach Thomas/Schaak Torsten/Triebl Katrin, Zwangsverheiratung in Deutschland. Anzahl und Analyse von Beratungsfällen, Opladen/ Berlin/Farmington Hills 2011.

5 Bericht „La prévalence du mariage forcé en Suisse: Rapport de l'enquête exploratoire», Fondation Surgir, Lausanne 2006, S. 11.

6 Neubauer Anna/Dahinden Janine, „Zwangsheiraten " in der Schweiz: Ursachen, Formen, Ausmaß, 2012, S. 2 f. Diese Untersuchung hat das "Maison d'analyse des processus sociaux“ MAPS der Universität Neuenburg im Auftrag des Bundes erstellt.

7 Strafbarkeit von Zwangsheiraten und arrangierten Heiraten: Bericht des Bundesrates in Erfüllung des Postulates 05.3477 der Staatspolitischen Kommission des Nationalrates vom 9.9.2005, S. 6 f.

8 Art. 14 der Bundesverfassung der Schweizerischen Eidgenossenschaft (BV, SR 101).

9 Siehe insbesondere Art. 23 Abs. 3 UNO-Pakt II sowie Art. 16 Abs. 1 lit. b des Übereinkommens zur Beseitigung jeder Form von Diskriminierung der Frau.

10 Art. 10 Abs. 2 BV.

11 Meier Yvonne, Zwangsheirat. Rechtslage in der Schweiz. Rechtsvergleich mit Deutschland und Österreich, Bern 2010, S. 6.

12 Ergebnisse einer Befragung zu dem Thema Zwangsheirat in Hamburg. Durchgeführt im Auftrag der Behörde für Soziales, Familie, Gesundheit und Verbraucherschutz in Hamburg. Johann Daniel Lawaetz-Stiftung. Bearbeitet: Thomas Mirbach/Simone Müller/Katrin Triebl, Oktober 2006, S. 25, Abbildung 3.3.2.

13 Ergebnisse einer Befragung zu dem Thema Zwangsheirat in Hamburg. Durchgeführt im Auftrag der Behörde für Soziales, Familie, Gesundheit und Verbraucherschutz in Hamburg. Johann Daniel Lawaetz-Stiftung. Bearbeitet: Thomas Mirbach/Simone Müller/Katrin Triebl, Oktober 2006, S. 72

14 Fachstelle für Gleichstellung, Stadt Zürich (Hrsg.), Zwangsheirat in Zürich, Hintergründe, Beispiele, Folgerungen, Zürich März 2010, Nachdruck Mai 2011, S. 15.

15 Fachstelle für Gleichstellung, Stadt Zürich (Hrsg.), Zwangsheirat in Zürich, Hintergründe, Beispiele, Folgerungen, Zürich März 2010, Nachdruck Mai 2011, S. 17 f.

16 Fachstelle für Gleichstellung, Stadt Zürich (Hrsg.), Zwangsheirat in Zürich, Hintergründe, Beispiele, Folgerungen, Zürich März 2010, Nachdruck Mai 2011, S. 18

17 Umfassend dazu Meier Yvonne, Zwangsheirat. Rechtslage in der Schweiz. Rechtsvergleich mit Deutschland und Österreich, Bern 2010 S. $12 \mathrm{ff}$. Zu den verschiedenen rechtlichen Antworten siehe Ziff. VI. und VII.
18 Ergebnisse einer Befragung zu dem Thema Zwangsheirat in Hamburg. Durchgeführt im Auftrag der Behörde für Soziales, Familie, Gesundheit und Verbraucherschutz in Hamburg. Johann Daniel Lawaetz-Stiftung. Bearbeitet: Thomas Mirbach/Simone Müller/Katrin Triebl, Oktober 2006, S. $51 \mathrm{ff}$

19 Ergebnisse einer Befragung zu dem Thema Zwangsheirat in Hamburg. Durchgeführt im Auftrag der Behörde für Soziales, Familie, Gesundheit und Verbraucherschutz in Hamburg. Johann Daniel Lawaetz-Stiftung. Bearbeitet: Thomas Mirbach/Simone Müller/Katrin Triebl, Oktober 2006, S. 58

20 Siehe dazu die Diskussion im Zusammenhang mit der schweizerischen Gesetzgebung Ziff. VI.

21 Siehe dazu Ziff. XI.

22 Postulat 05.3477 der Staatspolitischen Kommission des Nationalrats. Strafbarkeit von Zwangsheiraten und arrangierten Heiraten.

23 Motion 06.3658 Heberlein. Gesetzliche Maß nahmen gegen $Z$ wangsheiraten und arrangierte Heiraten.

24 Schweizerisches Zivilgesetzbuch vom 10. Dezember 1907 (ZGB, SR 210).

25 Bundesgesetz vom 18. Dezember 1987 über das Internationale Privatrecht (IPRG, SR 291).

26 Bundesgesetz vom 18. Juni 2004 über die eingetragene Partnerschaft gleichgeschlechtlicher Paare (Partnerschaftsgesetz, PartG, SR 211.231).

27 Bundesgesetz vom 16. Dezember 2005 über die Ausländerinnen und Ausländer (Ausländergesetz, AuG, SR 142.20).

28 Asylgesetz vom 26. Juni 1998 (AsylG, SR 142.31).

29 Schweizerisches Strafgesetzbuch vom 21. Dezember 1937 (StGB, SR 311.0).

30 BBl 2012 5937, wobei die Referendumsfrist am 4. Oktober 2012 ablief.

31 Neu Art. 99 Abs. 1 Ziff. 3 ZGB

32 Neu Art. 43 a Abs. $3^{\text {bis }}$ ZGB

33 Neu Art. 105 Ziff. 5 und 6 ZGB.

34 Neu Art. 106 Abs. 1 zweiter Satz ZGB.

35 Neu Art. 6 Abs. 1 und Art. 9 Abs. 1 Bst. d und e sowie Abs. 2 zweiter Satz PartG.

$36 \mathrm{Neu}$ Art. 45a IPRG.

37 Neu Art. 44 IPRG.

38 Art. 44 Abs. 2 IPRG wird aufgehoben, d.h. Eheschließungen in der Schweiz stehen ausschließlich unter schweizerischem Recht. Der neue Art. 45a IPRG stellt sicher, dass Art. 105 ZGB auch im internationalen Verhältnis greift. Siehe BBl $20112207 \mathrm{f}$.

39 Siehe oben.

$40 \mathrm{BBl} 20112210 \mathrm{f}$

41 Neu Art. 45a AuG sowie Art. 85 Abs. 8 AuG, neu Art. 51 Abs. 1 und $1^{\text {bis }}$ AsylG sowie Art. 71 Abs. 1 Einleitungssatz und $1^{\text {bis }}$ AsylG.

42 BBl 2011 2213; Amtliches Bulletin der Bundesversammlung, Nationalrat, Frühjahrsession 2012, 28. Februar 2012, S. 34 f.; Amtliches Bulletin der Bundesversammlung, Ständerat, Sommersession 2012, 5. Juni 2012, S. 448 f

43 BBl 2011 2213; Amtliches Bulletin der Bundesversammlung, Nationalrat, Frühjahrsession 2012, 28. Februar 2012, S. 31 f.

44 Siehe gleich anschließend.

45 Siehe dazu Ziff. XI.

46 Neu Art. 88a AuG sowie neu Art. 79a AsylG. 47 Neu Art. 181a StGB.

48 Amtliches Bulletin der Bundesversammlung, Nationalrat, Frühjahrsession 2012, 28. Februar 2012, S. 40.

49 Amtliches Bulletin der Bundesversammlung, Nationalrat, Frühjahrsession 2012, 28. Februar 2012, S. 38 f.

50 Motion 12.3097 Straftatbestand der Zwangheirat als Ausschaffungsgrund, eingereicht am 8. März 2012: „Der Bundesrat wird beaufragt, bei der Ausarbeitung der Umsetzung de Ausschaffungs-Initiative den Straftatbestand der Zwangsheirat in den Katalog der Ausschaffungsgründe aufzunehmen. " Siehe: http:// www.parlament.ch/d/suche/seiten/geschaefte. aspx?gesch_id=20123097 (zuletzt eingesehen am 4.10.2012).

51 Strafbarkeit von Zwangsheiraten und arrangierten Heiraten; Bericht des Bundesrates in Erfüllung des Postulates 05.3477 der Staatspolitischen Kommission des Nationalrates vom 9.9.2005, S. 2 (Hervorhebung durch Autorin weggelassen).

52 Stellungnahme von Amnesty International, Schweizer Sektion, vom 12. Februar 2009, zu den gesetzlichen Maßnahmen gegen Zwangsheiraten, siehe http://www.humanrights.ch/ upload/pdf/090215_AICH_Vernehmlassung Zwangsheirat.pdf (zuletzt eingesehen am 4.10.2012).

53 Stellungnahme des Vereins Humanrights.ch/ MERS vom 16. Februar 2009 zu den gesetzlichen Maßnahmen gegen Zwangsheiraten, siehe http://www.humanrights.ch/upload/ pdf/090216 Vernehmlassung_Zwangsheirat. pdf (zuletzt eingesehen am 4.10.2012).

54 Eidgenössisches Justiz- und Polizeidepartement EJPD, Bundesamt für Justiz BJ, 06.3658 Motion Heberlein. Gesetzliche Maßnahmen gegen Zwangsheiraten. Vorentwurf vom November 2008. Ergebnisbericht Oktober 2009, S. 6.

55 Stellungnahme der Eidgenössischen Kommission für Frauenfragen vom 22. Dezember 2008 , siehe http:/www.ekf.admin.ch/dokumentation/00441/index.html?lang=de (zuletzt eingesehen am 4.10.2012).

56 Medienmitteilung des Eidgenössischen Justizund Polizeidepartements vom 21. Oktober 2009, http://www.ejpd.admin.ch/content/ejpd/ de/home/dokumentation/mi/2009/2009-10-21. html (zuletzt eingesehen am 4.10.2012).

57 Insbesondere Schwander Marianne, Das Opfer im Strafrecht. Aktuelles und potenzielles Opfer zwischen Recht, Psychologie und Politik, Bern 2010, S. 99, mit weiteren Hinweisen.

58 Insbesondere Schwander Marianne, Das Opfer im Strafrecht. Aktuelles und potenzielles Opfer zwischen Recht, Psychologie und Politik, Bern 2010, S. 102, mit weiteren Hinweisen.

59 Insbesondere Schwander Marianne, Das Opfer im Strafrecht. Aktuelles und potenzielles Opfer zwischen Recht, Psychologie und Politik, Bern 2010, S. 103.

60 Insbesondere Schwander Marianne, Das Opfer im Strafrecht. Aktuelles und potenzielles Opfer zwischen Recht, Psychologie und Politik, Bern 2010, S. 104.

61 Medienmitteilung des Bundesrats vom 14. September 2012, siehe http:/www.ejpd.admin. ch/content/ejpd/de/home/dokumentation/ mi/2012/2012-09-14.html (zuletzt eingesehen am 4.10.2012).

62 Siehe Ziff, II, Fn. 6

63 Insbesondere Mirbach Thomas/Schaak Torsten/ Triebl Katrin, Zwangsverheiratung in Deutschland. Anzahl und Analyse von Beratungsfällen, Opladen/Berlin/Farmington Hills 2011, S. 25.

64 Westphal Manuela, Geschlechterstereotype und Migration, in: Bundesministerium für Familie, Senioren, Frauen und Jugend (Hrsg.), Forschungsreihe Band 1, Zwangsverheiratungen in Deutschland. Konzeption und Redaktion: Deutsches Institut für Menschenrechte, BadenBaden 2007, S. 131.

65 Häusliche Gewalt im Kanton Zürich. Evaluation der polizeilichen Schutzmaßnahmen im Kanton Zürich gemäß kantonalem Gewaltschutzgesetz für den Zeitraum der Inkraftsetzung des Gesetzes vom 1. April 2007 - 31. Dezember 2009, IST Interventionsstelle gegen Häusliche Gewalt/Direktion der Justiz und des Innern des Kantons Zürich (Auftraggeber), Jérome Endrass/Astird Rossegger/Frank Urbaniok (Verfasser des Berichts), 19. April 2012. 
66 Steiner Silvia, Häusliche Gewalt. Erscheinungsformen, Ausmaß und polizeiliche Bewältigungsstrategien in der Stadt Zürich 1999-2001, Zürich/Chur 2004, S. $121 \mathrm{f}$.

67 Meyer Albert, Die Verbrechen in ihrem Zusammenhang mit den wirtschaftlichen und sozialen Verhältnissen im Kanton Zürich, Jena 1895.

68 Zur Häuslichen Gewalt siehe umfassend Schwander Marianne, Das Opfer im Strafrecht. Aktuelles und potenzielles Opfer zwischen Recht, Psychologie und Politik, Bern 2010, S. $111 \mathrm{ff}$. mit weiterführenden Hinweisen.

69 Insbesondere Mirbach Thomas/Schaak Torsten/ Triebl Katrin, Zwangsverheiratung in Deutschland. Anzahl und Analyse von Beratungsfällen, Opladen/Berlin/Farmington Hills 2011, S. 26. Siehe auch Ziff. VIII.

70 Hirsi-Ali Ayaan, Victims of Islam: Women, International Humanist and Ethical Union, Juni 2005, siehe http://www.iheu.org/node/1534 (zuletzt eingesehen am 4.10.2012); Dies., Ich klage an. Plädoyer für die Befreiung der muslimischen Frau, München 2009.

71 Kelek Necla, Die fremde Braut. Ein Bericht aus dem Innern des türkischen Lebens in Deutsch- land, Köln 2005; Dies., Chaos der Kulturen. Die Debatte um Islam und Integration, Köln 2012

72 Mirbach Thomas/Schaak Torsten/Triebl Katrin, Zwangsverheiratung in Deutschland. Anzahl und Analyse von Beratungsfällen, Opladen/Berlin/Farmington Hills 2011, S. 27, mit weiterführenden Hinweisen.

73 Mirbach Thomas/Schaak Torsten/Triebl Katrin, Zwangsverheiratung in Deutschland. Anzahl und Analyse von Beratungsfällen, Opladen/Berlin/Farmington Hills 2011, S. 28.

74 Mirbach Thomas/Schaak Torsten/Triebl Katrin, Zwangsverheiratung in Deutschland. Anzahl und Analyse von Beratungsfällen, Opladen/Berlin/Farmington Hills 2011, S. 28.

75 Insgesamt Deutscher Bundestag Drucksache 17/4401, 17. Wahlperiode, 13.01.2011 Gesetzesentwurf der Bundesregierung Entwurf eines Gesetzes zur Bekämpfung der Zwangsheirat und zum besseren Schutz der Opfer von Zwangsheirat sowie zur Änderung weiterer aufenthalts- und asylrechtlicher Vorschriften, S. 2

76 BGBl. Nr. 33 vom 30.06.2011, S. 1266
77 \$237 Strafgesetzbuch Deutschland.

78 Insbesondere Terres des Femmes/Myria Böhmecke (Hrsg.), Im Namen der Ehre. Misshandelt. Zwangsverheiratet. Ermordet. Hilfsleitfaden für die Arbeit mit von Zwangsheirat/Gewalt im Namen der Ehre bedrohten und betroffenen Mädchen und Frauen, Tübingen 2007, S. 44 f.

$79 \$ 37$ (2) Gesetz über den Aufenthalt, die Erwerbstätigkeit und die Integration von Ausländern im Bundesgebiet (Aufenthaltsgesetz - AufenthG).

$80 \$ 1317$ (1) Bürgerliches Gesetzbuch (BGB).

$81 \int 8$ (3) Gesetz über den Aufenthalt, die Erwerbstätigkeit und die Integration von Ausländern im Bundesgebiet (Aufenthaltsgesetz - AufenthG).

$82 \int 88$ a Gesetz über den Aufenthalt, die Erwerbstätigkeit und die Integration von Ausländern im Bundesgebiet (Aufenthaltsgesetz - AufenthG).

83 Art. 1 ZwHeiratBekG Änderung des Aufenthaltsgesetzes mit Hinweis auf $\$ 61$ Abs. 1 Satz 3 .

\section{Paradoxien der Kriminalitätsfurcht}

\section{Welchen Stellenwert haben Kriminalitätsrisiken, Medienberichterstattung und generalisierte Ängste für die Veränderungen des lokalen Sicherheitsgefühls in der Bevölkerung?}

Karl-Heinz Reuband

\section{Einleitung}

Die jüngst veröffentlichten Zahlen des Bundeskriminalamtes für 2011 zeigen: Düsseldorf ist unter den deutschen Großstädten in der Kriminalitätsbelastung inzwischen auf Platz 2 aufgerückt. An erster Stelle steht nach wie vor Frankfurt mit 16.137 Delikten pro 100.000 Einwohner, gefolgt von Düsseldorf mit 15.130 und Köln mit 14.838. Dass Düsseldorf einen der Spitzenplätze in der Kriminalstatistik einnimmt, ist nicht neu: in den Jahren zuvor war Düsseldorf schon wiederholt an 3. oder 4. Stelle der unsicheren Städte platziert gewesen. Und wenn man den Zeitverlauf der letzten 20 Jahre betrachtet, kann man erkennen, dass die Stadt seit längerem - wenn auch nicht immer geradlinig - einen Anstieg in der Kriminalitätsbelastung durchlief. Demgegenüber stagnierten oder sanken die entsprechenden Zahlen auf bundesweiter Ebene (Bundeskriminalamt 1990ff.)

Die Spitzenpositionierung von Düsseldorf gilt nicht für alle Delikte. Im Hinblick auf die Delikte, die mit Gewalt verbunden sind und in der Bevölkerung als besonders bedrohlich angesehen werden, liegt Düsseldorf eher im oberen Mittelfeld. In Bezug auf Körperverletzung waren im Jahr 2010 Frankfurt oder Freiburg z.B. weitaus stärker belastet. Und im Fall der Raubdelikte auf Straßen, Wegen und Plätzen waren es Hamburg und Dortmund. Gleichwohl, was aus der Perspektive der Entwicklung hier von besonderem Interesse ist: in Düsseldorf sind auch die Delikte vom längerfristigen Anstieg betroffen, die mit Gewalt und Gewaltandrohung verbunden sind und die Bürger besonders beunruhigen.

So stieg die Häufigkeitszahl für „gefährliche und schwere Körperverletzung “ von 197 im Jahr 1998 auf 234 im Jahr 2004 und erreichte 2009 den Wert 254. Dies entspricht einem Anstieg von nahezu $30 \%$. Einen eher gebremsten Anstieg kennzeichnet die Entwicklung der Häufigkeitszahl für „Raubüberfälle auf Straßen, Wegen oder Plätzen “. Diese stieg von 83 im Jahr 1998 auf 112 im Jahr 2006. Seitdem ist sie wieder leicht gesunken und lag 2009 mit einem Wert von 81 praktisch auf dem gleichen Niveau wie 1998. Im Gegensatz zu diesen Delikten nahm die Häufigkeitszahl für Einbruch in Wohnungen bis vor kurzem kontinuierlich ab: von 530 im Jahr 1998 auf 384 im Jahr 2004 und schließlich auf 285 im Jahr 2007. Das entspricht nahezu einer Halbierung der Ausgangszahlen. Seit 2007 jedoch ist wieder ein Anstieg zu verzeichnen, 2009 belief sich die entsprechende Zahl auf 386 - was einem Zuwachs von rund einem Drittel entspricht.

Was bedeutet diese Entwicklung für das Kriminalitätserleben und damit auch für die Lebensqualität der Bürger? Spiegelt sich die überproportional hohe Kriminalitätsbelastung in einer ausgeprägten Kriminalitätsfurcht wider, leben viele in Angst und Schrecken und sind in ihrer Lebensführung massiv beeinträchtigt? Und ist die Furcht in all den Jahren, in denen die polizeiliche Kriminalstatistik hohe Zuwachsraten dokumentierte, parallel dazu gestiegen? Die Frage ist mehr als nur von lokalem Interesse, an ihr kann allgemein das Verhältnis von objektiver Bedrohung und Risiken auf 